\title{
Determination of Accessible Chloromethyl Groups in Chloromethylated Styrene-Divinylbenzene Copolymers
}

\author{
Viviane G. Teixeira ${ }^{a, b}$, Fernanda M. B. Coutinho ${ }^{*, a, c}$, Fernanda R. M. Petrocínio $^{a}$ \\ and Ailton S. Gomes ${ }^{a}$ \\ ${ }^{a}$ Instituto de Macromoléculas Professora Eloisa Mano, Universidade Federal do Rio de Janeiro, \\ CP 68525, 21945-970 Rio de Janeiro - RJ, Brazil \\ ${ }^{b}$ Departamento de Química, Instituto de Ciências Exatas, Universidade Federal Rural do Rio de Janeiro, \\ Seropédica - RJ, Brazil \\ ${ }^{c}$ Departamento de Processos Químicos, Instituto de Química, Universidade do Estado do Rio de Janeiro, \\ Rio de Janeiro - RJ, Brazil
}

\begin{abstract}
Este trabalho descreve uma avaliação do método de determinação de grupos clorometila em copolímeros de estireno-divinilbenzeno de diferentes tipos de porosidade baseado na reação de quaternização da piridina. Foram propostas algumas modificações no método descrito por Feinberg e Merrifield de acordo com o tipo de porosidade do copolímero a ser analisado. A reação de quaternização foi limitada pelo acesso da piridina aos grupos clorometila, que é determinado principalmente pelo inchamento do copolímero nesse reagente.
\end{abstract}

This paper describes an evaluation of the method based on the quaternization of pyridine for determining the chloromethyl groups content in styrene-divinylbenzene copolymers with different kinds of porosity. Some modifications of the method described by Feinberg and Merrifield are proposed depending on the porous characteristic of the copolymer to be analyzed. The quaternization reaction was limited by the access of pyridine to chloromethyl groups that is governed by the swelling equilibrium of the copolymer in the reactant.

Keywords: styrene-divinylbenzene copolymers, chloromethylation degree, pyridine, porosity

\section{Introduction}

Chemical modification of styrene-divinylbenzene (SDVB) copolymers by immobilization of chloromethyl groups is the first step in most of the routes used for functional copolymers preparation. ${ }^{1-3}$ The importance of this Friedel-Crafts alkylation is due to the generation of copolymers containing the very labile chlorine atom that permits many substitution reactions difficult to occur in the inert S-DVB copolymer. Several chloromethylation routes are described in literature and efforts have been made to avoid the use of the carcinogenics chloromethyl and bischloromethyl ethers, ${ }^{4-10}$ the two most classical chloromethylating agents. ${ }^{1-13}$ Wright $^{14}$ and coworkers published an interesting review on chloromethylation routes.

In order to evaluate any of these routes, chloromethylation degree is one of the most important

* e-mail: fern@uerj.br,fern@ima.ufrj.br parameters to be determined, allied to group distribution on the copolymer beads. For this purpose, elemental analysis is often used but this method is not so accurate for determining low chlorine contents. ${ }^{9,13}$ The titration of the burning products of copolymer after their absorption by a suitable solution is also used, but this method requires an appropriate apparatus, uncommon in most laboratories. ${ }^{15}$ Gravimetric determination after fusion of the copolymer with sodium peroxide is another method used by Pepper and coworkers, ${ }^{16}$ but many authors prefer the method based on the quaternization reaction of a tertiary amine by the copolymer. n-Butylamine has been used ${ }^{17,18}$ but pyridine is frequently preferred. ${ }^{12,13,19}$ After the quaternization reaction, the pyridinium chloride formed is titrated with standard silver nitrate solution. The end point is determined potentiometrically because no visual indicator can be used in this reaction medium. Elemental analysis and analysis of burning or fusion products are methods based on polymer matrix destruction and thus determine the total content of 
chloromethyl groups present in the sample. The quaternization reaction is a nondestructive method and only measures the amount of groups accessible to pyridine during the reaction. This information is frequently enough, specially when chloromethylation only constitutes a step in a synthetic route. In this case only those accessible groups will take part in the subsequent reaction. For qualitative evaluation of chloromethyl groups, Galindo et al. ${ }^{19}$ proposed a method based on the reaction with 4-(4nitrobenzyl)pyridine to form a coloured compound attached to the polymer matrix. The color intensity was used to verify the progress of chlorine substitution reactions.

Although the quaternization reaction of pyridine is well known for low molecular weight and soluble molecules, no attention has been given to its extent in different kinds of porous morphology of S-DVB copolymers. The same procedure is usually performed to determine chloromethyl group contents in gel-like and porous materials. But the process of reagent diffusion is particular for each kind of porous structure and will influence directly the accessibility of chloromethyl sites by reagents. ${ }^{20}$ Thus, the extent of quaternization reaction must be governed by the mass transfer process in each kind of porous morphology. In this work we tried to determine the best conditions for quantitative determination of chloromethyl groups by the method based on the quaternization of pyridine in different porous structures of S-DVB copolymers.

\section{Experimental}

\section{Chemicals}

Styrene (S), divinylbenzene (DVB) (both commercial grade, donated by Nitriflex Ind. Com.) and chloromethylstyrene (CMS) (Aldrich Chem. Co., 90\%) were washed with $5 \% \mathrm{~m} / \mathrm{m} \mathrm{NaOH}$ (Vetec Química Fina Ltda.) aqueous solution and neutralized with deionized water to eliminate inhibitor. 2,2'-Azobisisobutyronitrile (AIBN) (donated by Metacril S.A.) was recrystallized from methanol (Prosint). Poly(vinyl alcohol) (PVAL) (Hoechst, $88 \%$ hydrolysis degree), n-heptane, N,Ndimethylformamide, nitric acid (all purchased from Vetec Química Fina Ltda.), silver nitrate and pyridine (both from Merck), all of P.A. grade, were used as received.

\section{Instruments}

Potentiometric titrations of chloride ions were performed using an Analyser 6A09 oxidation-reduction combined electrode with silver ring adapted to an Analyser 300M potentiometer. A Perkin Elmer 1720X Infrared Spectrophotometer was used to record spectra of terpolymers as $\mathrm{KBr}$ discs. Surface area of the terpolymers was measured by applying BET method to nitrogen adsorption in a Micromeritics ASAP 2010 apparatus. Optical appearance was observed in an Olympus SZ10 optical microscope.

Styrene-divinylbenzene-chloromethylstyrene (S-DVBCMS) terpolymers synthesis

S-DVB-CMS terpolymers were synthesized by aqueous suspension polymerization using a 4:1 ratio between aqueous and organic phases. The aqueous phase was constituted of $\mathrm{NaCl} 0.5 \% \mathrm{~m} / \mathrm{v}$, to diminish the monomer solubility in water, and PVAL $0.5 \% \mathrm{~m} / \mathrm{v}$, as suspension agent. After prepared, the aqueous phase was transferred to a $1 \mathrm{~L}$ three-necked round bottomed flask fitted to a condenser. The system was mechanically stirred at a rate of $350 \mathrm{rpm}$. The organic phase, consisting of a S/DVB/ CMS mixture $(25 / 65 / 10$ or $57 / 39 / 4$ molar ratios), AIBN $1 \%$ molar of total monomers and $n$-heptane in varied proportions, as pore forming agent, was also added to the flask and kept at $75^{\circ} \mathrm{C}$ under stirring for $24 \mathrm{~h}$. The beads obtained were washed several times with hot water and finally sieved. The 45-80 mesh fraction was separated and washed with hot ethanol. After the purification process, the terpolymer was imbibed with methanol and dried from this solvent for $48 \mathrm{~h}$ at $60{ }^{\circ} \mathrm{C}$.

Porous structure characterization of S-DVB-CMS terpolymers

Porous structure of S-DVB-CMS terpolymer was characterized by determining: apparent density by the graduated cylinder method $;{ }^{21}$ fixed pore volume by water uptake measurements; ${ }^{22}$ surface area by nitrogen adsorption, by applying BET method, and optical appearance by optical microscopy. Swelling properties of the terpolymers were evaluated in toluene, n-heptane and pyridine. $^{23}$

\section{Determination of accessible chloromethyl group content in S-DVB-CMS terpolymers}

The experimental procedure followed the method proposed by Feinberg and Merrifield, ${ }^{12}$ but some parameters were changed in our study, as will be described in results and discussion section. In this general procedure, $0.5 \mathrm{~g}$ of resin was weighed in the test tube and $4 \mathrm{~mL}$ of 
pyridine were added. The tube was then stoppered and heated to $100{ }^{\circ} \mathrm{C}$ for $1 \mathrm{~h} 30 \mathrm{~min}$. When the system attained room temperature, $6 \mathrm{~mL}$ of $\mathrm{N}, \mathrm{N}$-dimethylformamide (DMF) and $6 \mathrm{~mL}$ of concentrated nitric acid were added. The tube was cooled again to room temperature in an ice bath. The tube content was quantitatively transferred to a beaker with DMF and potentiometrically titrated with standard silver nitrate solution. An oxidation-reduction combined electrode with a silver ring sensible to chloride ions was used. Second derivative method was employed to determine the end point volume, thus no potentiometer calibration was needed.

\section{Results and Discussion}

\section{Porous structure characterization of $S-D V B-C M S$ terpolymers}

S-DVB-CMS terpolymers were synthesized in presence of n-heptane as pore forming agent. In the synthesis of copolymers VT-25 to VT-27 (see Table 1), three dilution degrees were adopted with the aim of producing different types of porous structures. No changes were made in other parameters, such as DVB content or type of diluent, to attain this objective. This decision was based on the fact that these changes would promote more differences in the process of porous structure formation of the terpolymer during its synthesis. Thus, the association of these parameters would determine the porous structure formed and the comparison between them could become more difficult. As shown in Table 1, a terpolymer with a gel-like structure was produced when $50 \%$ dilution degree was used (VT-25). This structure is characterized by very low values of surface area (not measurable by BET method) and fixed pore volume, associated to a high apparent density. As the amount of n-heptane, a poor solvent for the copolymer, was increased (dilution degree), structures with permanent porosity were formed, as can be noted by the lower apparent density values of VT-26 and VT-27 terpolymers. These materials have also significant values of surface area and fixed pore volume. One can observe that VT-26 (100\% dilution degree) has a higher surface area than VT-27 (200\% dilution degree), what suggests the enhancement of average pore diameter as the volume of n-heptane was increased in the polymerization medium.

The difference between the swelling degrees in toluene and n-heptane decreases when dilution degree increases. This behavior indicates that the amount of gel phase in copolymers VT-26 and VT-27 is smaller than in VT-25 and so a permanent porosity (fixed pores) is present in these materials.

In order to evaluate the influence of crosslinking degree on the determination of chloromethyl groups, a terpolymer with higher DVB content was synthesized (VT-28). This polymer has a porosity similar to VT-26 as can be seen by the values of apparent density, fixed pore volume and specific surface area. Therefore, its polymer matrix is more rigid, what is demonstrated by the low swelling degree in toluene and n-heptane.

Figure 1 shows the optical appearance of S-DVB-CMS terpolymers. VT-25, the gel-like one, is translucent, VT-26 is white and bright and VT-27 is white and opaque. Opacity increases as light diffraction occurs more intensely, due to the higher porosity of the polymer beads. These materials were used to evaluate the method for determining the chloromethylation degree.

\section{Quantitative determination of chloromethyl groups}

Four terpolymers of S-DVB-CMS with different kinds of porous structure were produced, but the theoretical content of chloromethylated monomer was assumed to be the same in VT-25, VT-26 and VT-27, because the proportion of chloromethylstyrene in the monomer mixture was kept constant. VT-28 was synthesized by using a lower proportion of CMS in the monomer mixture. Consequently, these materials can be used as standards of chloromethylated S-DVB copolymers in order to establish a quantitative methodology of chloromethyl group determination in different types of porous structures, since their porosity and the content of chloromethyl groups are known. Considering the total incorporation of monomers and the $90 \%$ purity of chloromethylstyrene used in the synthesis, the theoretical value of chloromethyl group

Table 1. Porous characteristics of S-DVB-CMS terpolymers

\begin{tabular}{|c|c|c|c|c|c|c|c|c|}
\hline Terpolymer & $\begin{array}{l}\mathrm{DD}^{\mathrm{a}} \\
(\%)\end{array}$ & $\begin{array}{l}\text { S/DVB/CMS } \\
\text { molar ratio }\end{array}$ & $\begin{array}{c}\mathrm{d}_{\mathrm{a}}^{\mathrm{b}} \\
\left(\mathrm{g} \mathrm{cm}^{-3}\right)\end{array}$ & $\begin{array}{c}\mathrm{V}_{\mathrm{fp}}^{\mathrm{c}} \\
\left(\mathrm{cm}^{3} \mathrm{~g}^{-1}\right)\end{array}$ & $\begin{array}{c}\mathrm{S}^{\mathrm{d}} \\
\left(\mathrm{m}^{2} \mathrm{~g}^{-1}\right)\end{array}$ & $\begin{array}{c}\mathrm{I}_{\mathrm{tol}}^{\mathrm{e}} \\
(\% \mathrm{v} / \mathrm{v})\end{array}$ & $\begin{array}{c}\mathrm{I}_{\mathrm{hep}}{ }^{\mathrm{f}} \\
(\% \mathrm{v} / \mathrm{v})\end{array}$ & $\begin{array}{c}\mathrm{I}_{\mathrm{py}}^{\mathrm{g}} \\
(\% \mathrm{v} / \mathrm{v})\end{array}$ \\
\hline VT-25 & 50 & & 0.59 & 0.03 & $\mathrm{~nm}^{\mathrm{h}}$ & 70 & 45 & 64 \\
\hline VT-26 & 100 & $65 / 25 / 10$ & 0.34 & 0.77 & 116 & 53 & 40 & 57 \\
\hline VT-27 & 200 & & 0.18 & 1.92 & 41 & 36 & 21 & 36 \\
\hline VT-28 & 100 & $57 / 39 / 4$ & 0.32 & 0.92 & 139 & 30 & 23 & 26 \\
\hline
\end{tabular}

a Dilution degree with n-heptane; ${ }^{\mathrm{b}}$ apparent density; ${ }^{\mathrm{c}}$ fixed pore volume; ${ }^{\mathrm{d}}$ surface area; ${ }^{\mathrm{e}} \mathrm{swelling}$ degree in toluene; ${ }^{\mathrm{f}}$ swelling degree in n-heptane; ${ }^{g}$ swelling degree in pyridine; ${ }^{\mathrm{h}}$ not measurable by BET method. 
a)

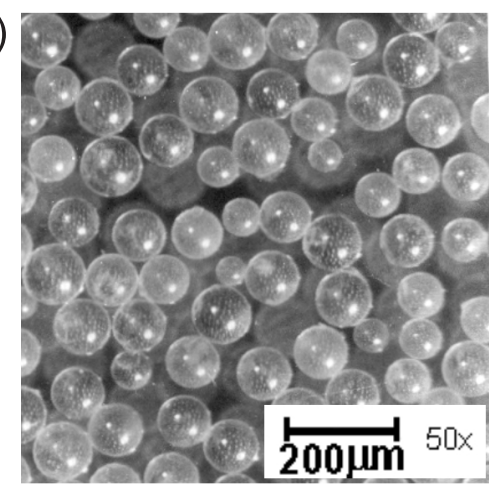

b)

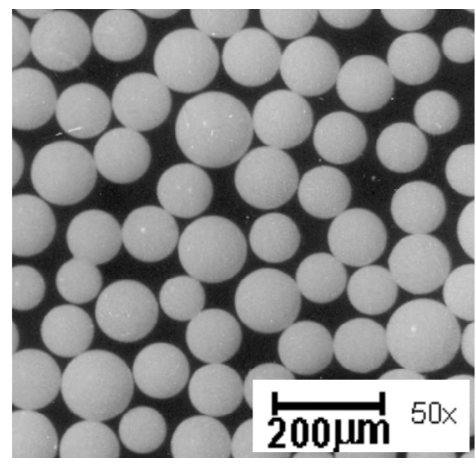

c)

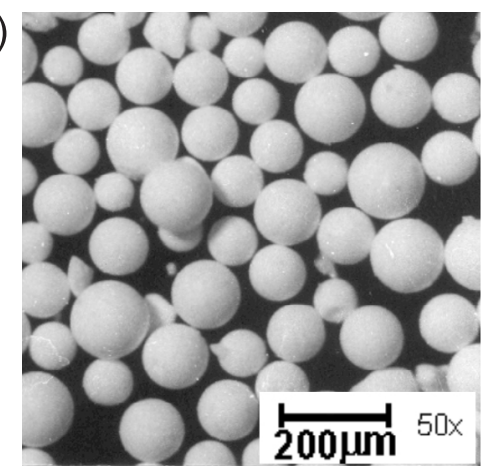

Figure 1. Optical micrographies of VT-25 (a), VT-26 (b) and VT-27 (c)

content in the terpolymers is $0.786 \mathrm{mmol}-\mathrm{CH}_{2} \mathrm{Cl} \mathrm{g}^{-1}$. This value is believed to be the maximum content of chloromethyl groups possible to be achieved in a quantitative determination.

The presence of chloromethyl groups was confirmed by FTIR spectroscopy. The presence of absorption band at $1266 \mathrm{~cm}^{-1}$ due to $\mathrm{CH}_{2}-\mathrm{Cl}$ stretching was used as a qualitative indication of the presence of these groups.

In the general procedure for quantitative determination of accessible chloromethyl groups described by Feinberg and Merrifield, the polymer is weighed and readily reacted with pyridine. The extent of this reaction is dependent on the pyridine access to chloromethyl sites, what is governed by its diffusion through the bulk of the material and by the swelling equilibrium of this material in the reactant. As shown in Table 1 concerning terpolymers swelling degrees, pyridine is a solvent for the terpolymer as good as toluene is, and the latter is known to be a good solvent for S-DVB copolymers. Rabelo and Coutinho ${ }^{23}$ defined the uptake of toluene by a macroporous S-DVB copolymer as a result of three contributions: filling of fixed pores, pore expansion and nuclei swelling. Therefore, these processes will also govern the access of pyridine to chloromethyl groups of a macroporous material. In a gel type material, the uptake of a good solvent is promoted by the expansion of polymer chains, what makes the reactivity of this kind of material more dependent on its swelling properties in the reactant medium, since there is no contribution of fixed pore filling. That is, the surface area in a gel type polymer is lower than in macroporous one, where the readly available amount of chloromethyl groups is higher. These considerations and the results of chloromethyl groups content determination allowed us to believe, by using Feinberg and Merrifield method, that a previous step of polymer sample swelling in pyridine could be necessary. The swelling permits the reactant to reach the more internal groups and consequently the reaction will be more complete, especially for gel type polymers. Table 2 shows the results of chloromethyl group content achieved by following the method described by Feinberg and Merrifield and the results achieved after some modifications introduced by us in the method. A difference between theoretical and experimental chloromethyl group contents was observed for all terpolymers studied. Probably, this difference (error) was also associated to the assumption that $100 \%$ of monomers were incorporated during terpolymer synthesis. Higher values of error were observed for VT-27 and VT-28. Adversely, VT-25, the gel type terpolymer, showed the lower error value, followed by VT-26. A $3 \mathrm{~h}$ reaction was carried out with VT-25 and VT-26 to evaluate if the time reaction adopted, $2 \mathrm{~h}$, was enough to complete the quaternization reaction. That could be a limiting factor, enhancing the analysis error. But no significant differences were observed, leading to the conclusion that $2 \mathrm{~h}$ is time enough to complete the quaternization reaction of accessible groups.

Trying to explain the high error values of these determinations, a $24 \mathrm{~h}$ swelling step in pyridine before heating was introduced in the original methodology. It produced a decrease in error values as observed in Table 2. Thus, a second swelling step was introduced. The terpolymer already reacted with pyridine was swollen in the $\mathrm{HNO}_{3} / \mathrm{DMF}$ mixture during $24 \mathrm{~h}$ before potentiometric titration of chloride ions. As this mixture is not a good solvent for the terpolymer, this step is not a true swelling one, but the establishment of a new diffusion equilibrium condition after the addition of $\mathrm{HNO}_{3} / \mathrm{DMF}$ mixture to the system. The high polarity of this mixture difficults its 
Table 2. Results of chloromethyl group content determinations in S-DVB-CMS terpolymers

\begin{tabular}{|c|c|c|c|c|}
\hline Parameter & VT-25 & VT-26 & VT-27 & VT-28 \\
\hline S/DVB/CMS molar ratio $(\%)$ & & $65 / 25 / 10$ & & $57 / 39 / 4$ \\
\hline Dilution degree (n-heptane) (V/V,\%) & 50 & 100 & 200 & 100 \\
\hline Theoretical $-\mathrm{CH}_{2} \mathrm{Cl}$ content $\left(\mathrm{mmol} \mathrm{g}^{-1}\right)$ & & 0.786 & & 0.301 \\
\hline \multirow[t]{3}{*}{$-\mathrm{CH}_{2} \mathrm{Cl}$ content $2 \mathrm{~h}$ reaction $\left(\mathrm{mmol} \mathrm{g}{ }^{-1}\right)$} & 0.702 & 0.684 & 0.535 & 0.181 \\
\hline & $\Delta^{\mathrm{a}}=0.003$ & $\Delta=0.002$ & $\Delta=0.002$ & $\Delta=0.003$ \\
\hline & $\delta^{\mathrm{b}}=-11 \%$ & $\delta=-13 \%$ & $\delta=-32 \%$ & $\delta=-39 \%$ \\
\hline \multirow[t]{3}{*}{$-\mathrm{CH}_{2} \mathrm{Cl}$ content $3 \mathrm{~h}$ reaction $\left(\mathrm{mmol} \mathrm{g} \mathrm{g}^{-1}\right)$} & 0.675 & 0.689 & & \\
\hline & $\Delta=0.03$ & $\Delta=0.01$ & - & - \\
\hline & $\delta=-14 \%$ & $\delta=-12 \%$ & & \\
\hline \multirow[t]{3}{*}{$-\mathrm{CH}_{2} \mathrm{Cl}$ content $\mathrm{S}_{\mathrm{py}}{ }^{\mathrm{c}}\left(\mathrm{mmol} \mathrm{g}^{-1}\right)$} & 0.727 & 0.724 & 0.539 & 0.216 \\
\hline & $\Delta=0.01$ & $\Delta=0.07$ & $\Delta=0.01$ & $\Delta=0.01$ \\
\hline & $\delta=-8 \%$ & $\delta=-8 \%$ & $\delta=-31 \%$ & $\delta=-28 \%$ \\
\hline \multirow{3}{*}{$-\mathrm{CH}_{2} \mathrm{Cl}$ content $\mathrm{S}_{\mathrm{py}, \mathrm{HNO}, \mathrm{DMF}}{ }^{\mathrm{d}}\left(\mathrm{mmol} \mathrm{g}^{-1}\right)$} & 0.727 & 0.708 & 0.577 & 0.217 \\
\hline & $\Delta=0.008$ & $\Delta=0.04$ & $\Delta=0.01$ & $\Delta=0.002$ \\
\hline & $\delta=-8 \%$ & $\delta=-10 \%$ & $\delta=-27 \%$ & $\delta=-28 \%$ \\
\hline
\end{tabular}

Number of determinations for each sample $(\mathrm{n})=2$. a Difference between two determinations; ${ }^{\text {error }}$ between theoretical and experimental values of $-\mathrm{CH}_{2} \mathrm{Cl}$ content; ${ }^{\mathrm{c}}$ swelling in pyridine; ${ }^{\mathrm{d}}$ swelling in pyridine followed by swelling in $\mathrm{HNO}_{3}$ and DMF; (-) not determined.

diffusion through out the polymer matrix and then the chloride ions produced in the inner parts of polymer may not be titrated. The results shown in Table 2 led us to the conclusion that this equilibrium condition was the same even after $24 \mathrm{~h}$ of contact. The chloromethyl group content did not change with the addition of this step, except for VT-27 where a smaller difference was observed. This terpolymer has the highest value of fixed pore volume and a low surface area, what suggests a material with high average pore diameter. The diffusion of the polar mixture must be easier through this porous structure, making this step important for this kind of material. Maybe, no diffusion else occurred in the other structures, due to their lower average pore diameter.

Comparing all the results of chloromethyl groups content determination for the four structures, it is clear that swelling in pyridine was responsible for diminishing the difference between theoretical and experimental values of chloromethyl groups. For VT-28 the decreasing was more accentuated than for the other terpolymers. The error diminishment for VT-25 and VT-26 was lower and no change was observed for VT-27. This behavior and the fact that VT-25 and VT-26 presented the lowest values of error can be explained with the aid of swelling degrees shown in Table 1. VT-25 and VT-26 present the highest values of swelling degree in pyridine. The swelling equilibrium condition for these materials is rapidly achieved and even chloromethyl groups present in the gel phase become accessible to pyridine. The low surface area of VT-25 does not influence the access of pyridine to chloromethyl groups, because its high swelling capacity compensates this characteristic. As expected, no error diminishment was attained with the step of swelling in $\mathrm{HNO}_{3} / \mathrm{DMF}$ mixture for VT-25 and VT-26, what can be explained by the very low affinity between solid and liquid phases. Comparing
VT-27 and VT-28, both have comparable swelling behaviors in all solvents used, but VT-28 was synthesized with a higher amount of DVB than the other terpolymers. The swelling in pyridine was more effective for the determination of chloromethyl group content of VT-28 than for the one of VT-27. These two terpolymers are the most entangled ones. VT-28 presents an entangled structure due to its higher degree of crosslinking and VT-27, due to the higher dilution degree employed in its synthesis. The higher degree of crosslinking makes VT-28 a more rigid structure than VT-27 and the swelling equilibrium condition must be achieved latter by VT-28 than by VT27 , even though both at the end achieve the same equilibrium condition. For VT-28, no enhancement in cloromethyl group content was observed with the swelling in $\mathrm{HNO}_{3} / \mathrm{DMF}$ mixture. This material presents values of surface area and fixed pore volume similar to those of VT26. Consequently, its average pore diameter is also low and does not permit solution diffusion through out the structure. The rigid structure of VT-28 presents two disadvantages: it has a limited swelling in pyridine and the size of its pores is very small to permit satisfactory diffusion of $\mathrm{HNO}_{3} / \mathrm{DMF}$ mixture. The association of these two factors enhances the difference between experimental and theoretical values.

\section{Conclusions}

The determination of chloromethyl groups in chloromethylated S-DVB copolymers by the method envolving a quaternization reaction of pyridine must follow a specific methodology for each kind of porous structure. These methodologies must be defined based on the establishment of the swelling equilibrium condition of each structure in the reactant, pyridine, and on the 
establishment of $\mathrm{HNO}_{3} / \mathrm{DMF}$ mixture diffusion through the polymer matrix. Chloromethyl group determination in gel structures may be carried out readily after the addition of pyridine because swelling equilibrium condition is rapidly attained. Structures with high crosslinking degree need a swelling step in pyridine while structures with high average pore diameter permits the inclusion of a swelling step in $\mathrm{HNO}_{3} / \mathrm{DMF}$ mixture.

This method only determines the amount of chloromethyl groups accessible to pyridine. Because of this characteristic, it fits well on chloromethyl groups determinations in materials submitted to chloromethylation reaction after their synthesis. In these materials, all immobilized chloromethyl groups are located in regions accessible to pyridine (a good solvent for the copolymer) and consequently they will be determined.

\section{Acknowledgements}

Conselho Nacional de Desenvolvimento Científico e Tecnológico (CNPq), Programa de Apoio ao Desenvolvimento Científico e Tecnológico (PADCT/ CNPq), Fundação de Amparo à Pesquisa do Estado do Rio de Janeiro (FAPERJ), Nitriflex and Metacril for their support of this work.

\section{References}

1. Merrifield, R. B.; J. Am. Chem. Soc. 1963, 85, 2149.

2. Dalal, M. K.; Ram, R. N.; J. Mol. Catal. A: Chem. 2000, 159, 285.

3. Patel, S. A.; Sinha, S.; Mishra, A. N.; Kamath, B. V.; Ram, R. N.; J. Mol. Catal. A: Chem. 2003, 159, 53.

4. Olah, G. A.; Beal D. A.; Yu, S. H.; Olah, J. A.; Synthesis 1974, 560 .
5. Olah, G. A.; Beal, D. A.; Yu, S. H.; Olah, J. A.; J. Org. Chem. 1976, 4, 1976.

6. Warshawsky, A.; Deshe, A.; J. Polym. Sci.: Polym. Chem. 1985, 23, 1839.

7. Ye C.; Marks, T. J.; Yang, J.; Wong, G. K.; Macromolecules 1987, 20, 2324.

8. Itsuno, S.; Uchikoshi, K.; Ito, K.; J. Am. Chem. Soc. 1990, 112,8187

9. Avram, E.; Luca, C.; Polym.-Plast. Technol. Eng. 1996, 35, 757.

10. DeHaan, F. P.; Djaputra, M.; Grinstaff, M. W.; Kaufman, C. R.; Keithly, J. C.; Kumar, A.; Kuwayama, M. K.; Macknet. K. D.; Na, J.; Patel, B. R.; Pinkerton, M. J.; Tidwell, J. H.; Villahermosa, R. M.; J. Org. Chem. 1997, 62, 2694.

11. Fuson, R. C.; Mckeever, C. H. In Organic Reactions; Adams, R. ed.; John Wiley \& Sons, Inc.: New York, 1942, p. 63B90.

12. Feinberg, R. S.; Merrifield, R. B.; Tetrahedron 1974, 30, 3209.

13. Li, R.; Xiao, X. Y.; Czarnik, A. W.; Tetrahedron Lett. 1998, 39 , 8581.

14. Wright, M. E.; Toplikar, E. G.; S. Svejda, A.; Macromolecules 1991, 24, 5879.

15. Haslam, J.; Hamilton, J. B.; Squirrell, D. C. M.; Analyst 1960, $85,556$.

16. Pepper, K. W.; Paisley, H. M.; Young, M. A.; J. Am. Chem. Soc. 1953, 4097.

17. Petit, M. A.; Jozefonvicz, J.; J. Appl. Polym. Sci. 1977, 21, 2589.

18. Okay, O.; Die Angew. Makromol. Chem. 1986, 143, 209.

19. Galindo, F.; Altava, B.; Burguete, M. I.; Gavara, R.; Luis, S. V.; J. Comb. Chem. 2004, 6, 859.

20. Veverka, P.; Jerábek, K.; React. Funct. Polym. 1999, 41, 2.

21. Sherrington, D. C.; Nouv. J. Chem. 1982, 6, 12, 661.

22. Rabelo, D.; Coutinho, F. M. B.; Polym. Bull. 1993, 30, 725.

23. Rabelo, D.; Coutinho, F. M. B.; Eur. Polym. J. 1994, 30, 6, 675. 